\title{
JPL's MEMS Gyroscope Fabrication, 8-Electrode Tuning, and Performance Results
}

\author{
Sam Y. Bae, Ken J. Hayworth, Kirill V. Shcheglov, Karl Y. Yee, and Dean V. Wiberg. \\ Jet Propulsion Laboratory \\ Pasadena, CA 91109 \\ http://mems.jpl.nasa.gov
}

\begin{abstract}
In this paper, we present JPL's 8-electrode MEMS gyroscope, which allows for full electrostatic tuning of the resonant structure. It has shown a sufficient electrostatic tuning range needed to fully correct the native frequency mismatches of our current fabrication process. Test data shows that this gyroscope design has Q's averaging 30,000 (measured via ringdown time method) and native frequency mismatch on the order of $0.4 \%$. The electrostatic tuning of the mismatched frequencies has successfully demonstrated $0.02 \%$ matching for the test batch and down to $0.003 \%(10 \mathrm{mHz}$ at $3000 \mathrm{~Hz})$ repeatable frequency matching during specific tuning experiments. As a result, we can report angle random walk (ARW) value of $0.1 \mathrm{deg} / \mathrm{rthr}$ and $2 \% \mathrm{hr}$ bias instability (Allan variance $1 /$ f floor estimated). A third party, Honeywell Inc., tested one of the earlier designed 4-electrode gyroscopes, which achieved frequency degeneracy through a mechanical ion-milling of the springs, and reported $1 \%$ hr bias instability.
\end{abstract}

\section{INTRODUCTION}

The Jet Propulsion Laboratory (JPL) has an ongoing effort to develop a silicon based miniaturized vibratory gyroscope for space navigation applications. Manufacturing of the gyroscope follows established standard technologies used in the silicon batch fabrications of MEMS devices, permitting the gyroscope to be batch-produced, thereby available to a larger market at lower cost. At the same time, the JPL gyroscope has incorporated features designed to stretch its performance to a navigation-qualified sensor as described in previous publications. [1, 2]

Figure 1 is a SEM photo of a JPL MEMS gyroscope. Figure 2 delineates its components.

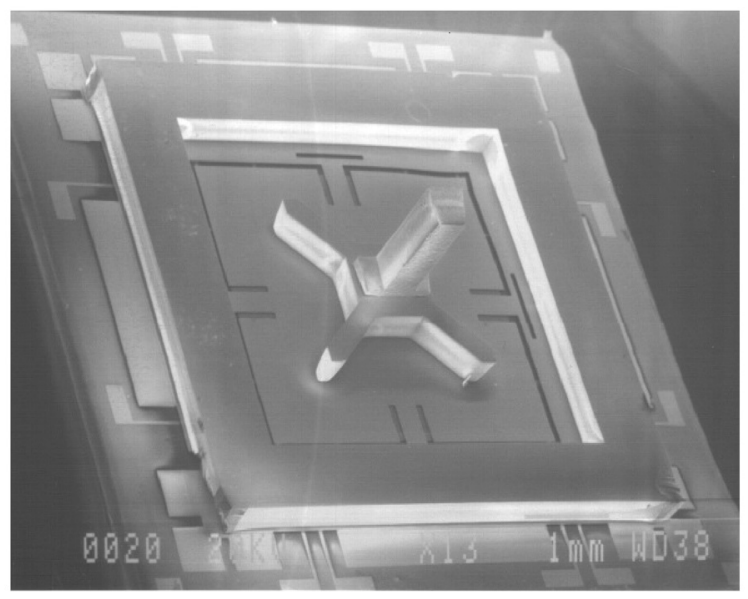

Figure 1. (Left) An SEM photo of a fabricated JPL MEMS gyroscope

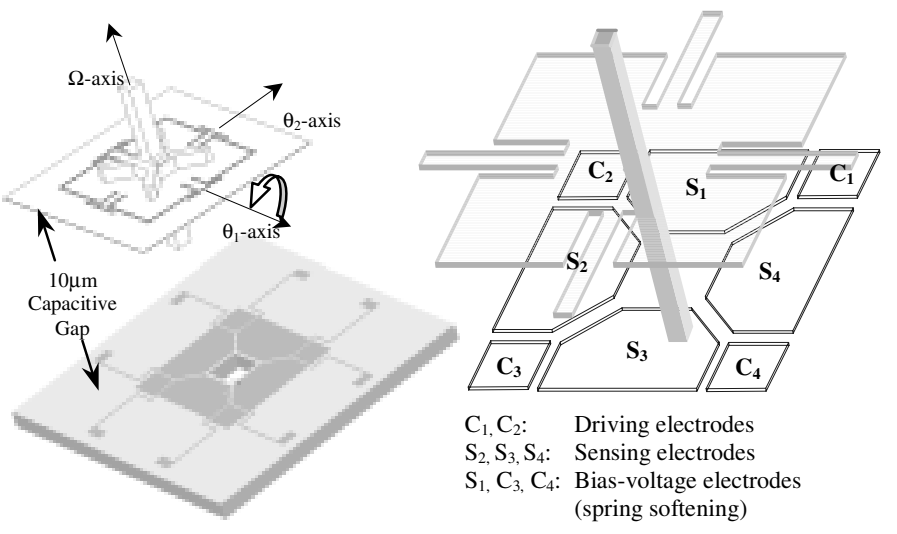

Figure 2. (Left) A sketch of JPL MEMS gyroscope's cloverleaf (top) and electrode-plate (bottom) (Right) 8-Electrode layout. 4petal membrane drawn semi-transparently on top of the electrodes.

A gyroscope consists of three parts: 1) a two degree-offreedom 4-petal clover membrane, suspended by two-fold symmetric $4 \mathrm{~T}$-springs at each mid-edge, 2) a post, the main inertial element, runs perpendicular to $\theta_{1}-\theta_{2}$ plane (hereon we call the first two components the "cloverleaf"), 3) 8-electrode plates that drive and sense the capacitive changes of the cloverleaf's membrane (hereon call the "electrode-plate"). The total dimensions of the assembled MEMS gyroscope are $9 \times 9 \times 6 \mathrm{~mm}$.

The 4-petal clover membrane 2 degrees-of-freedom which exhibit two closely matched natural modes. These have the shape of plate rocking modes. While a positive voltage is applied to the cloverleaf, Electrodes $\mathrm{C}_{1}$ and $\mathrm{C}_{2}$ (Figure 2 right) drive the cloverleaf's 4-petal membrane sinusoidally with respect to the $\theta_{1}$ axis to one of the rocking modes (this is the "drive-mode"). When the gyroscope rotates around the $\Omega$-axis, the energy in the drivemode is transferred to the other rocking mode, the "sense-mode," inducing a skew motion with respect to the $\theta_{2}$-axis. Then, the Electrodes $S_{2}, S_{3}$, and $S_{4}$ pick up the capacitive changes, and attached transimpedance amplifiers convert this varying capacitance into a voltage. Finally, the sense signal is demodulated by multiplying with the drive signal; the demodulated signal produces a baseband signal proportional to the inertial rate of the gyroscope in the direction of the $\Omega$-axis. In practice, an automatic gain control (AGC) loop tracks the natural frequency of the gyroscope, and a force rebalance (FRB) loop damps the transient energy transferred to the sense-mode, ensuring fast and stable rate response of the overall signal. The rest of the electrodes, $\mathrm{C}_{3}, \mathrm{C}_{4}$, and $\mathrm{S}_{1}$, are used for electrostatic tuning; properly biasing the three with respect to the resonator causes the unmatched frequencies to become closely matched and aligned to the preferred modal axis by softening the $4 \mathrm{~T}$-springs. (See 
Electrostatic Tuning Section) In the perfect case, the rocking modes have two equal frequencies (i.e. degenerate, $\mathrm{f}_{1}=\mathrm{f}_{2}$ ) and only Q and electronic noise would limit the performance. Note in many other gyroscope designs, the springs are either trimmed or ablated mechanically through a laser or ion-etcher to achieve modal degeneracy; however, the JPL gyroscope's $10 \mu \mathrm{m}$ gap and large electrode area allow simple electrostatic tuning to replace the mechanical tuning of gyroscopes.

\section{FABRICATIONS}

Electrode-plate fabrication starts with a $500 \mu \mathrm{m}$ thick, $100 \mathrm{~mm}$ diameter, (100) oriented, double side polished, resistance as low as $10 \Omega$-cm and P-type doped wafer. Cloverleaf fabrication start with a $26 \mathrm{micron}$ thin SOI, $500 \mu \mathrm{m}$ thick, $100 \mathrm{~mm}$ diameter, double side polished, resistant as low as $10 \Omega$-cm wafers and p-type doped wafer.

Table 1. Cross-sections of the JPL MEMS gyroscope fabrications.

\section{Electrode-plate \\ Cloverleaf}

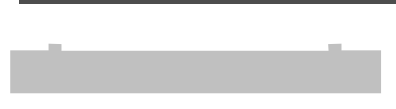

a. 8micron Pillar Etch
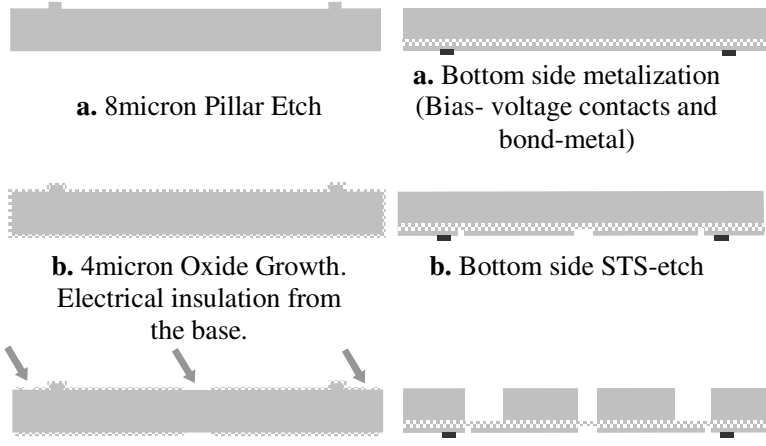

c. Oxide Openings

(Indicated with arrows)

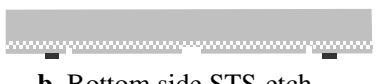

b. Bottom side STS-etch

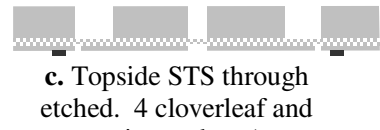
springs release)

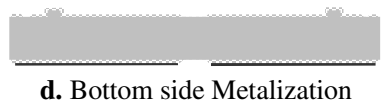
(Bond-metal)

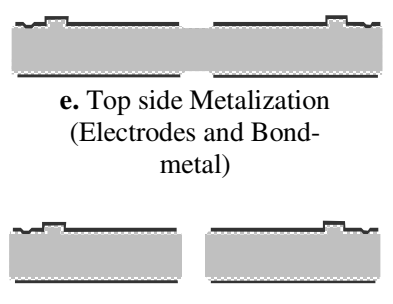

f. STS Through Etch

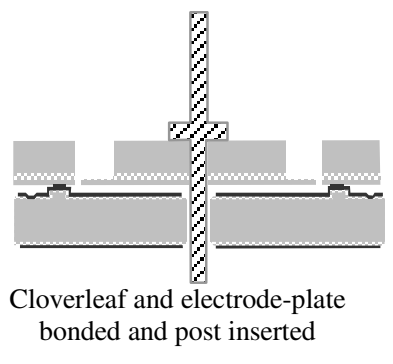

Performance Tuning

The performance of a vibratory gyroscope depends crucially on the physical properties of the resonant structure. In our case, the gyroscope performs optimally when 1) the 4-petal membrane has low dampening (high Qs), 2) the two rocking modes are closely matched in frequency, and 3) the first two properties are stable over both time and temperature [3].

To achieve high Q's, we chose a ceramic substrate mount (Figure 3 ) that provides a relatively large mass for stable and rigid mounting and tested in a low vacuum environment $\left(<10^{-3}\right.$ Torr $)$. Operation in vacuum is required because this type of large electrode area, a thin gap resonator is highly damped by squeeze film dampening in air. A rigid mounting to the package was required since the JPL gyroscope's cloverleaf was not balanced in its rocking modes unlike balanced tuning fork gyro designs. In other words, a fraction of the energy of a rocking mode contained in the package was subject to varying loses to the environment. The current substrate helped resolve this problem by brut force, the ratio of energies being proportional to the ratio of resonator mass to package mass. As a result, we attained Qs as high as 76,000. The Qs were measured by noting the ring down time of an undriven mode with no electronics attached.

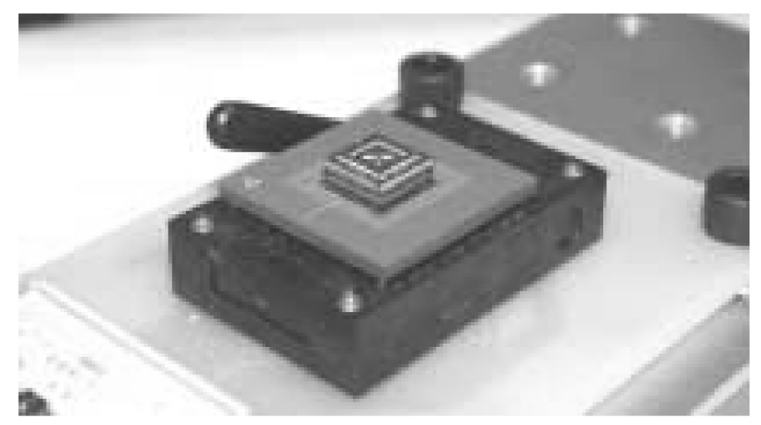

Figure 3. JPL MEMS Gyroscope mounted on a ceramic substrate then on zero-insertion-force socket (standard quad package).

\section{Electrostatic Tuning}

Like all other Coriolis force based gyroscopes, when the gyroscope is first assembled, imperfections in fabrications result in mismatched frequencies. (See Table 2 for statistics) The mismatched frequency was mainly due to uneven stresses received from a thermal compression bonding. The top of figure 4 shows a gyroscope having an $18.75 \mathrm{~Hz}$ frequency split, a $0.6 \%$ mismatch, and would have resulted in poor gyroscope performance or not even operating control loops. The bottom of figure 4 shows the same gyroscope after DC voltages had been placed on the Electrodes $\mathrm{C}_{3}, \mathrm{C}_{4}$, and $\mathrm{S}_{1}$. The modes were then split only by $200 \mathrm{mHz}$. (Later, we had gone further narrowing the splits to zero.) This condition is called modal degeneracy. At this state, the resonator behaves like a Foucault pendulum with no preferred rocking directions, and this gives maximum sensitivity of the device.

The gyroscope's resonator can be modeled as a 2 degree-offreedom torsional oscillator. Its rocking-mode is characterized by inertia, dampening, and stiffness matrices. The asymmetries in the inertia and stiffness matrices determine the frequency mismatch. The electrostatic tuning is based on the non-linear force equation applied in a biased capacitor. Applying this principle, we were then able to soften the springs of the resonator utilizing an ideal proportional feedback loop. Using a minimum of two bias electrodes, we aligned the stiffness matrix with the inertia matrix's asymmetries, thereby giving rise to the degenerate modes. We derived a set of tuning equations using a redundant set of 4 bias electrodes. We then implemented these non-linear equations to create three virtual "tuning knobs," using a DSP program. These "knobs" separate the tuning process into three parts: knob\#1) alignment of modes, knob\#2) setting of frequency f1, knob\#3) setting of frequency $\mathrm{f} 2$. Each of these steps is completely separate from the others and can thus be automated into control loops. Tuning of a gyroscope is thus accomplished by driving the 
resonator in a particular direction and tweaking Knob\#1 until only one natural mode is excited, as known as "quadrature nulling."

The drive loop is now locked onto driving only a single mode with frequency f1. Knob\#2 is now tweaked to set 11 to a desired target frequency. Finally, the drive control loop is switched to driving the orthogonal rocking direction, locking onto that mode at frequency f2. Knob\#3 is now tweaked to set $\mathrm{f} 2$ also to the target frequency. Because knob \#1,\#2 and \#3 are implemented to simultaneously change voltages on all 4 bias pads using the non-linear tuning equations, they ensure that the tweaking of knob\#3 only affects $\mathrm{f} 2$ and does not disturb $\mathrm{f} 1$ or modal alignment.

This technique was not fully developed at the time of testing the 7 gyroscopes in Table 2, which is why they have tuned frequencies listed that are still relatively large. We now run all our gyroscope with tuned splits in the range of $10 \mathrm{mHz}$ and are developing algorithms that will continuously tune our gyroscope during operation using the virtual "tuning knobs" described above. This will eventually allow for automatic frequency tuning compensating for time and temperature effects.
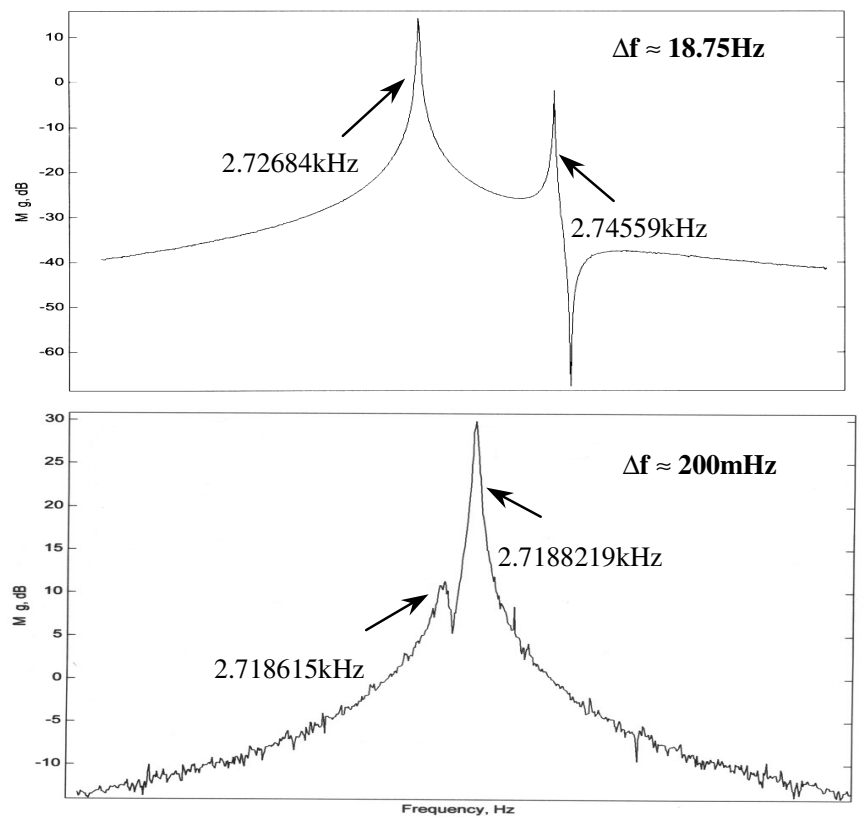

Figure 4. Micro-gyroscope \#8.

(Top) Native frequency split at rocking mode, $\Delta \mathrm{f} \approx$ $18.75 \mathrm{~Hz}$

(Bottom) After applying electrostatic bias voltages on 3 of the 8 electrodes, $\Delta \mathrm{f} \approx 200 \mathrm{mHz}$

\section{RESULTS}

As shown in Table 2, we tested 7 gyroscopes in house. The measured bias instability performance for each is given in the table. Rate table testing statistics for one gyrocope (gyroscope \#8) is shown in Figure 5. These include a rate linearity and repeatability test over the $\pm 1 \% \mathrm{sec}$ range, a plot of rate bias and rate drift over a 10 minute period, and a standard gyroscope Green chart analyzing this 10 minutes of drift data for its standard deviation statistics at various integration times. The bias instability figure is approximated as the minimum on this graph and the ARW as $1 / 60$ the value of the initial $-1 / 2$ slope line at 1 second integration time. Statistics for this particular gyroscope are $2 \% \mathrm{hr}$ bias instability and $0.1 \mathrm{deg} / \mathrm{rthr}$ ARW.
Table 2. Test results of the 7 JPL MEMS Gyroscopes

\begin{tabular}{|l|c|c|c|c|c|}
\hline Gyro & $\begin{array}{c}\mathbf{Q} \\
\text { (high) }\end{array}$ & $\begin{array}{c}\mathbf{Q} \\
(\mathbf{l o w})\end{array}$ & $\begin{array}{c}\text { Unmatched } \\
\text { Native } \\
\text { Frequency }\end{array}$ & $\begin{array}{c}\text { Tuned } \\
\text { frequency } \\
(\Delta \mathbf{f}=\mathbf{f 1 - f 2})\end{array}$ & $\begin{array}{c}\text { Perfor- } \\
\text { mance } \\
\text { \%/hr. }\end{array}$ \\
\hline 5 & 32,000 & 4,700 & 8.30 & 1.0 & 5 \\
\hline 6 & 19,000 & 9,300 & 8.50 & 1.0 & 2.0 \\
\hline 8 & 76,000 & 42,000 & 18.8 & 0.20 & 2.0 \\
\hline 9 & 30,000 & 12,000 & 10.6 & 0.50 & 25 \\
\hline 13 & 48,000 & 29,000 & 8.00 & 0.50 & 6.0 \\
\hline 14 & 19,000 & 17,000 & 12.0 & 0.26 & 10 \\
\hline 18 & 60,000 & 54,000 & 11.0 & 0.50 & 2.0 \\
\hline avg. & 41,000 & 24,000 & 11.0 & 0.57 & 10 \\
\hline
\end{tabular}

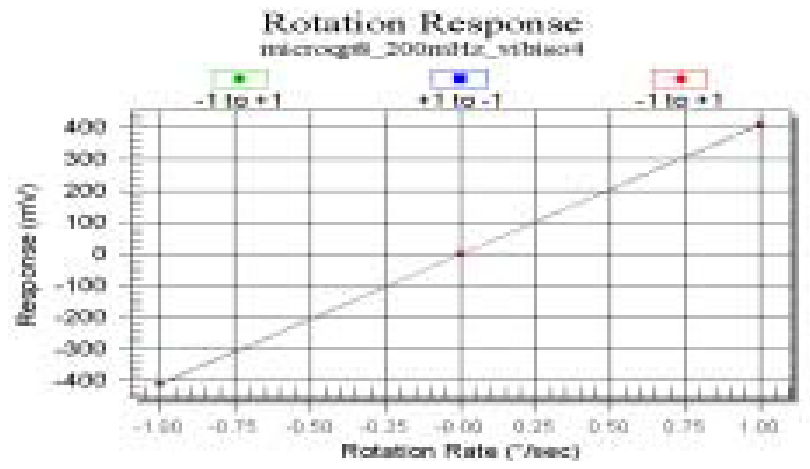

Drift

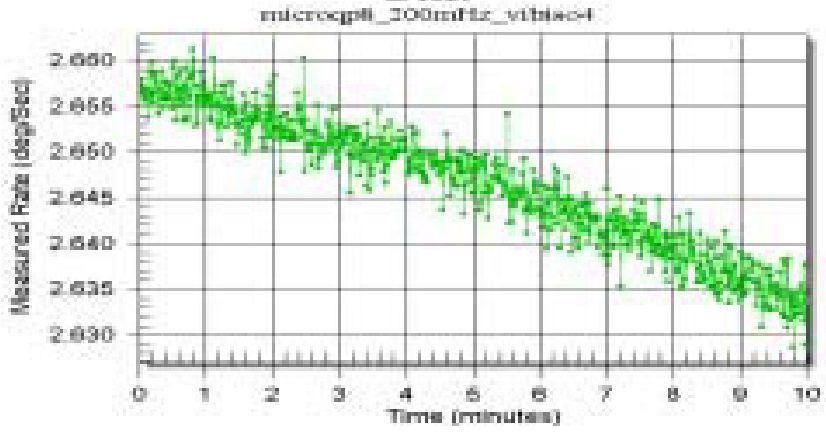

Green Chart

nuicrupps_200mitz_vibimo4: Reap $=411.1571$ deg/wes

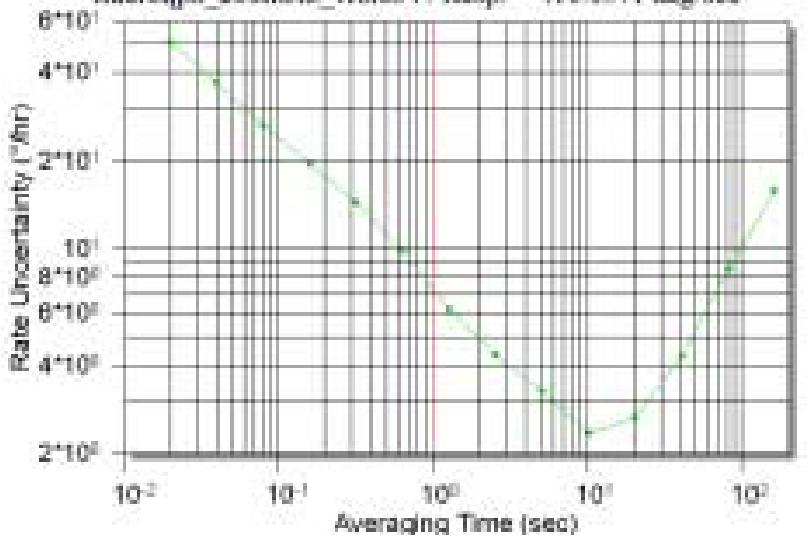

Figure 5. MEMS gyroscope \#8 (Top) Rotation response (Middle) Drift data (Bottom) Green chart; 
Figure 6 shows a Green Chart measured by a third party test of one of our gyro designs. Honeywell Inc. tested a 4-electrode gyroscope, not having extra biasing electrodes seen in 8-electrode gyroscope, and reported bias instability (flicker floor estimate) of $1 \% \mathrm{hr}$.

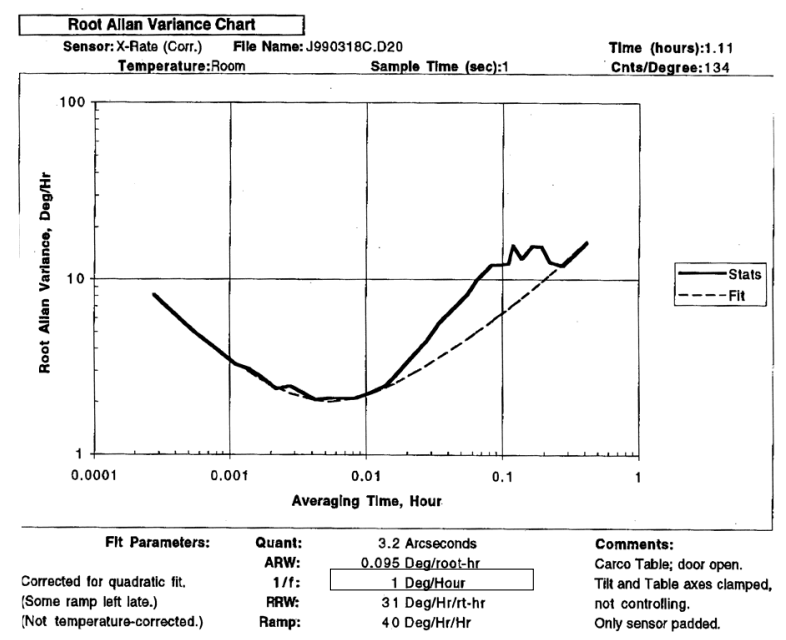

Figure 6. Honeywell Inc. tested a JPL micro-gyroscope, reported bias instability (1/f floor fit) of 1degree/hour (boxed)

\section{CONCLUSIONS}

Several unique attributes of the JPL gyroscope design account for its high performance marks. First, it is manufactured using a bulk silicon process with relatively large dimensions giving it high relative manufacturing precision. This also means the resonator (minus the post) is made of a single piece of high $Q$ silicon. Second, the resonator wafer is wafer bonded to the electrode wafer giving a thin, repeatable 10um capacitive gap with large surface area for sensing, driving, and electrostatic tuning of the resonator. Third, the symmetric electrode design and large electrostatic tuning range in the device, combined with the native precision above, allows for tuning to modal degeneracy in a majority of devices in a wafer. Fourth, the large inertial mass of the vertical post provides strong Coriolis coupling between these degenerate modes. These combined attributes have lead to a sensitive rate sensor.

\section{ACKNOWLEDGEMENT}

Funding for this work was provided by the Jet Propulsion Laboratory, Center for Integrated Space Microsystems and The National Aeronautics and Space Administration (NASA) Code R, UPN 755.

The JPL MEMS Team would like to thank Mr. Dorian Challoner for his full support during this project and valuable comments reflecting his in-depth knowledge of the subject.
[1] T. K. Tang, R. C. Gutierrez, J. Wilcox, C. Stell, V. Vorperian, R. Calvet. W. Li, I. Charkaborty, R. Bartman, W. Kaiser, "Silicon Bulk Micromachined Vibratory Gyroscope", Tech Digest, Solid-State Sensor and Actuator Workshop, Hilton Head, S. C. pp. 288-293, June 1996

[2] T. K Tang, R. C Gutierrez, C. B. Stell, V. Vorperian, G. A. Arakaki, J. T. Rice, W. J. Li, I. Chakrabory, K. Shcheglov, J. Z. Wilcox, W. J. Kaiser, "A packaged silicon MEMS vibratory gyroscope for microspacecraft", in Proc. IEEE Micro Electro Mechanical Systems Workshop (MEMS '97), Japan, 1997, pp. 500-505

[3] K. Shcheglov, C. Evans, R. Gutierrez, T. K. Tang, "Temperature dependent characteristics of the JPL silicon MEMS gyroscope", in Proc. IEEE Aerospace Conference 2000, Volume 1, pp. 403-411, 